\title{
High-Frequency Modeling and Filter Design for PWM Drives with Long Cables
}

\author{
Lei Wang ${ }^{1}$, Yifan Zhang ${ }^{2}$, Muhammad Saqib Ali ${ }^{1}$, Guozhu Chen ${ }^{1, *}$, Josep M. Guerrero ${ }^{3, *}$ (i) \\ and Juan C. Vasquez ${ }^{3}$ (D) \\ 1 College of Electrical and Engineering, Zhejiang University, Hangzhou 310027, China; \\ leiwang_ee@zju.edu.cn (L.W.); msaqibali@zju.edu.cn (M.S.A.) \\ 2 Polytechnic Institute, Zhejiang University, Hangzhou 310027, China; 21760552@zju.edu.cn \\ 3 Center for Research on Microgrids (CROM), Department of Energy Technology, Aalborg University, \\ 9220 Aalborg, Denmark; juq@et.aau.dk \\ * Correspondence: gzchen@zju.edu.cn (G.C.); joz@et.aau.dk (J.M.G.)
}

check for updates

Citation: Wang, L.; Zhang, Y.; Ali, M.S.; Chen, G.; Guerrero, J.M.; Vasquez, J.C. High-Frequency Modeling and Filter Design for PWM Drives with Long Cables. Energies 2021, 14, 1155. https://doi.org/ $10.3390 /$ en14041155

Academic Editor: Anna Rita Di Fazio

Received: 19 January 2021

Accepted: 16 February 2021

Published: 22 February 2021

Publisher's Note: MDPI stays neutral with regard to jurisdictional claims in published maps and institutional affiliations.

Copyright: (C) 2021 by the authors. Licensee MDPI, Basel, Switzerland. This article is an open access article distributed under the terms and conditions of the Creative Commons Attribution (CC BY) license (https:/ / creativecommons.org/licenses/by/ $4.0 /)$.

\begin{abstract}
Aiming at the problems emerging in a pulse width modulation (PWM) drive system with long cables, the accurate modeling of power cables is the premise for predicting and analyzing these relevant phenomena, and a proper filter design is the key solution to these problems. This paper proposes high-frequency cable models to represent these frequency-dependent characteristics, especially for the high-frequency resistance of cables, which is an easily overlooked factor that determines the damping of overvoltage. The proposed models can be used for accurately representing the cable characteristics in a wide frequency range, and correctly simulating the differential mode (DM) overvoltage and common mode (CM) current, including the peak value, oscillation frequency, and damping of the transient waveform. In addition, improved filter networks are proposed to suppress the DM voltage and CM current, with the merit of low losses, small volume, and excellent abilities for suppressing overvoltage. The proposed cable models and the filter design were validated in a $750 \mathrm{~W}$ PWM drive system with $200 \mathrm{~m}$ power cables.
\end{abstract}

Keywords: cable modeling; motor drives; filter design; DM overvoltage; CM current; PWM

\section{Introduction}

The pulse width modulated voltage source inverter (PWM-VSI) plays an important role in the adjustable speed drive (ASD) system, leading to more effective drive applications. New power semiconductor devices with high switching speed improve the power density of ASD applications [1], but electromagnetic interference (EMI) issues related to high frequency have emerged. The high-frequency problems will be more serious if long power cables are required to be connected between the inverter and motor, like the ASDs in offshore or land-based wells [2], mining plants [3], and wind farms [4], because the voltage reflection owing to cable-motor impedance mismatch occurs along the cable and twice or higher dc-bus voltage appears at the motor terminal, which may destroy the insulation of the cables and motors. In addition, high $\mathrm{dv} / \mathrm{dt}$ applied on the parasitic capacitance of a cable will produce high-frequency current, possibly resulting in the malfunction of the drive system. Apart from the insulation problem of motors, the generation of bearing current caused by a high dv/dt will shorten the motor lifetime [5]. To investigate and solve the problems of EMI and overvoltage, accurate modeling of the drive system and an elaborated filter design were the research objectives.

In the modeling of the long-cable drive system, the cable model is the most crucial part, and should be consistent with the practical high-frequency response. Certainly, researchers have also studied at full steam trying to establish accurate models for the inverter and motor, which are the other two parts of a simplified drive system. The inverter is often regarded as a voltage source with a trapezoidal or parabolic shape, and 
internal impedance may sometimes be considered. Various high-frequency models have been suggested for the motor [6-10], to represent the high-frequency characteristic of motors. Characterizing each phase of motors with a single circuit [6-8] is a universal highfrequency model, when the motor windings of every phase are symmetrical and balanced. Additionally, the rational function is used to fit the measured motor parameters $[9,10]$ proposes a motor model for both the time domain and frequency domain. As for cable models, it is known that the low frequency model is not adequate for analyzing the highfrequency phenomenon occurring in the drive system with long cables. References [11,12] applied mathematical models to fit the cable parameter dependency to frequency, but this is of a rather abstract mathematical derivation. Cable modeling in the frequency domain is another method worth exploring [13], which has both the advantage of short simulation time and the disadvantage of difficulty in constructing the inverter or harmonic voltage source. The lumped-parameter model has been widely adopted, including multi-order per section [14,15], and the second-order section [16], if classified by the section orders of the model. Although the model based on the time domain characteristics is widely accepted, the modeling process of a multi-order model with a higher accuracy is a little complicated. Generally, it is hard to find a modeling method including both simplicity and accuracy simultaneously. In addition, researchers almost always pursue the accuracy of cable impedance but neglect the resistance, which is a minor part of high-frequency impedance but plays an important role in simulating high-frequency phenomenon, leading to inaccuracy in simulating the overvoltage and CM current.

The passive filter is an effective method to solve the high-frequency problems in the long-cable drive system, and is easier to realize compared with insulation enhancement of motors or modulation improvement of the inverter control. The conventional LR, RLC, and RC filters are widely used as differential mode (DM) filters [17-19], and their parameter calculation is summarized in [19]. However, there are resistors in those filters as impedance matching components and power-consuming components, contributing to the low efficiency when used in a low-power drive system. At the same time, common mode (CM) noise should also be suppressed with a corresponding CM filter, and it was integrated as one filter with the DM filter in [20], which is hard to design and manufacture. Reference [21] proposed an innovative CM transformer whose magnetic core is smaller than that of the conventional CM choke, and which has been adopted in this paper.

In this paper, two high-frequency cable models were proposed to improve the frequencydependent characteristics of a power cable, and both models were compared, showing that the proposed ladder circuit has similar and even better performance, but takes more time to calculate and simulate. After the evaluation of the proposed model parameters with the analytical design equations provided, the entire cable model was constructed, and its validity and effectiveness were verified through using practical $200 \mathrm{~m}$ power cables. In order to check the simulation results of DM overvoltage and CM current based on the model of the drive system, including long cables, inverter, and motor model, an experiment was carried out on a $750 \mathrm{~W}$ test platform. Apart from the comparison between simulation results of the proposed model and experimental results, other cable models available in some software programs were also compared. Finally, the new filter networks, especially for a small hp motor with a strict working voltage requirement and long leads, were proposed to suppress the DM overvoltage and CM current. The advantages of the proposed filter network, including volume, efficiency, and suppression ability for overvoltage, were also confirmed by an experiment on the same test platform.

\section{High-Frequency Modeling of the Power Cables}

Accurate modeling of the power cables plays the most important role in predicting overvoltage and spike currents, and optimizing the filter design. It is known that the distributed-parameter model is normally used to represent the characteristics of cables, and is derived from the differential equation of the transmission line, but it does not include the frequency-dependent nature of the cable parameters, especially in the high-frequency 
range. On the contrary, the lumped-parameter circuit, which has the merits of flexibility and relatively easy realization, could provide such frequency-dependent characteristics once the lumped segment is accurate and its amount is adequate. Certainly, the most basic and conventional PI model, shown in Figure 1a, as a lumped-parameter circuit, is not enough to display frequency-dependent characteristics, but serves as the basis of most improved distributed-parameter models, which comprise series and parallel branches.

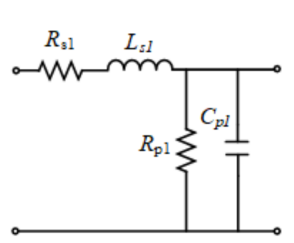

(a)

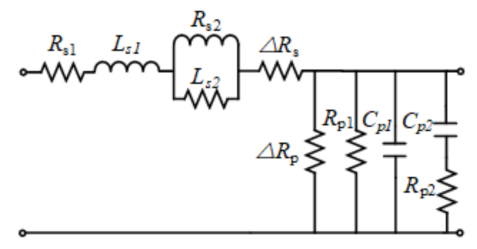

(b)

Figure 1. Elementary cell of the cable model.

Frequency-dependent phenomenon are mainly caused by dielectric losses, skin, and proximity effects appearing in the high-frequency range, resulting in the variation of cable parameters with frequency. In detail, the series inductance and parallel capacitance only have a slight decrease as frequency increases [17], showing an inverse correlation with frequency, but the series resistance instead increases sharply and nonlinearly as frequency increases. There is a similar phenomenon that exists in parallel resistance, which makes a small contribution to the cable characteristic because of its enormous value of resistance. For the convenience of representing these high-frequency characteristics, the impedance performance of a cable is usually selected as the evaluation index that actually only represents a part of cable characteristics, but researchers pay much attention to improved models that make the fitted impedance superimpose well onto the measured ones $[19,22]$. When the frequency increases, the inductance will dominate the impedance, while at the same time the resistance changes fast, but is much smaller than inductance, resulting in the enormous fitting deviation of high-frequency resistance; although with a good agreement with impedance performance. In other words, the good agreement between fitting and measured impedance only indicates that the inductance or capacitance of the model is consistent with the that of the cable in the high-frequency range, without considering the consistence of the series and parallel resistance at high frequency.

To fit the practical high-frequency characteristics of cable series resistance, as well as inductance, the R-L ladder circuit is introduced, as shown in Figure $2 \mathrm{a}$, consisting of $\mathrm{R}$ and $\mathrm{L}$ elements that are independent of frequency as a single element, but that contribute to the frequency-dependent characteristics of cable series resistance and inductance as a whole. Similarly for parallel resistance and capacitance, the R-C ladder circuit is introduced. The more branches of the ladder network, the higher the apparent fitting accuracy, but the more complicated the parameter calculation and the model simulation. As a matter of a fact, the parameter calculation of a ladder network with only three or four branches is still not easy, since it includes complex numbers and many variables, though some mathematical simplifications can be conducted in the calculation process [14]. The three and four branch R-L ladder networks, with parameters in Tables 1 and 2 are derived from corresponding sets of impedance at different frequency points in Tables 3 and 4, and are constructed to fit the curve of series resistance versus frequency, as shown in Figure 3, and the simulation results of both ladders are in good agreement. Better fitting performance for inductance was realized that is not shown here, because the variation of inductance with frequency is not very distinct and easier to fit, and so is the admittance, which will all be discussed afterwards. However, there should be a trade-off between the accuracy of the fitting series, parallel resistance, and the cost of modeling. It is known that the cable parameters should be evaluated over the frequency spectrum of voltage pulse, from several hundred hertz to several megahertz, but the overvoltage at the motor terminal rings at 
the same frequency as the natural frequency of the cable, decided by the cable length and its intrinsic characteristics. The natural frequency $\left(f_{R}\right)$ of the cable is a function of cable inductance per unit length $\left(L_{0}\right)$, cable capacitance per unit length $\left(C_{0}\right)$ and cable length $(l)$, further defined using cable length and relative permittivity $\left(\varepsilon_{r}\right)$, and can be expressed as:

$$
f_{R}=\frac{1}{4 l \sqrt{L_{0} C_{0}}}=\frac{c}{4 l \sqrt{\varepsilon_{r}}}
$$

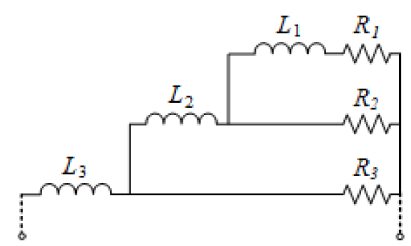

(a) R-L ladder,

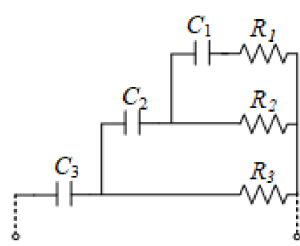

(b) R-C ladder.

Figure 2. Elementary cell of the ladder network.

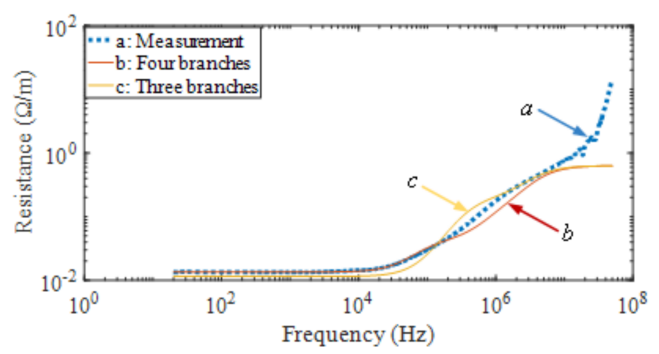

Figure 3. Per unit cable resistance of the measurement and simulation.

Table 1. Parameters of three branches.

\begin{tabular}{ccc}
\hline Branch & Resistance $(\boldsymbol{\Omega})$ & Inductance $(\mathbf{H})$ \\
\hline 1 & -0.0021 & $6.4444 \times 10^{-8}$ \\
2 & 0.1981 & $3.6495 \times 10^{-8}$ \\
3 & 0.6156 & $-4.3040 \times 10^{-8}$ \\
\hline
\end{tabular}

Table 2. Parameters of four branches.

\begin{tabular}{ccc}
\hline Branch & Resistance $(\Omega)$ & Inductance $(\mathbf{H})$ \\
\hline 1 & $-6.9474 \times 10^{-5}$ & $6.4723 \times 10^{-8}$ \\
2 & 0.0401 & $-3.5474 \times 10^{-8}$ \\
3 & 0.2443 & $3.2018 \times 10^{-8}$ \\
4 & 0.6156 & $-4.3040 \times 10^{-8}$ \\
\hline
\end{tabular}

Table 3. Measured points for three branches.

\begin{tabular}{ccc}
\hline Frequency $\mathbf{( H z )}$ & Impedance $(\boldsymbol{\Omega})$ & Angle $\left(^{\circ}\right)$ \\
\hline 28,710 & 0.0698 & 80.71 \\
$1.78 \mathrm{M}$ & 3.7289 & 87.19 \\
$5.01 \mathrm{M}$ & 10.2518 & 88.13 \\
\hline
\end{tabular}

Table 4. Measured points for four branches.

\begin{tabular}{ccc}
\hline Frequency $\mathbf{( H z )}$ & Impedance $(\Omega)$ & Angle $\left(^{\circ}\right)$ \\
\hline 28,710 & 0.0698 & 80.71 \\
$167 \mathrm{k}$ & 0.3802 & 85.83 \\
$1.78 \mathrm{M}$ & 3.7289 & 87.19 \\
$5.01 \mathrm{M}$ & 10.2518 & 88.13 \\
\hline
\end{tabular}


The cable resistance at the oscillation frequency, almost fixed for a specific cable from (1), plays an important role in the overvoltage simulation, and determines the decay speed or damping of the overvoltage. In addition, although the ladder network fits the characteristics of the cable well, it takes much effort to construct and still cannot guarantee the high accuracy of cable series and parallel resistance at the oscillation frequency. It cannot be denied that the ladder circuit with more branches is an effective model, if the simulation and calculation time are not taken into consideration. Therefore, on the other hand, the requirements of cable modeling can be changed as the consistency of the inductance and capacitance in the whole frequency range, but resistance consistency at natural frequency $\left(f_{R}\right)$ and some resistance inconsistency permitted at other frequencies. Then, an improved model (Figure 1b) was proposed based on a basic PI cable model, in which parallel branches $\left(R_{p 2}-C_{p 2}\right)$ represent the dielectric loss, and fit the capacitance and parallel resistance characteristics varying with frequency; series branch $\left(R_{s 2}-L_{s 2}\right)$ denotes the skin and proximity effects, and fits the inductance and series resistance characteristics varying with frequency; and the supplementary branch $\left(\Delta R_{S}-\Delta R_{p}\right)$ is used for the modification of the series and parallel resistance at the cable resonance frequency. Furthermore, the proposed model is available in both DM and CM equivalent circuits, only with parameter differences. In a typical inverter-fed motor drive, there are two phases in parallel and another phase in return between the inverter and motor, as the DM equivalent circuit of the cable, modelled to simulate the overvoltage phenomenon at the motor side. As for CM equivalent circuit, it consists of three phase lines in parallel and a ground line as a returning cable, used to analyze the CM noise of a long cable system. For the disambiguation of the following sections, the proposed model means the cable model in Figure $1 \mathrm{~b}$ rather than the ladder network in Figure 2.

\section{Parameter Evaluation of the Proposed Cable Model}

\subsection{Parameter Calculation}

The parameters of the proposed model could be determined by the short circuit (SC) and open circuit (OC) impedance of the power cables, which could be evaluated by an impedance analyzer. For the purpose of getting exact cable parameters, the length of the test cable was critical for the impedance measurement, and there should be no resonance in its impedance characteristic, meaning the cable should be short enough, but the measurement of short cables can be affected by the instrument's intrinsic deviation or the fixture impedance. Therefore, it is better to find a long cable with no resonance in the test frequency range. For convenience, the natural frequency of the cable could be roughly regarded as the first resonance frequency of cable impedance, then if the maximum measurement frequency is $f_{\max }$, the length of the test cable $\left(l_{\text {test }}\right)$ can be determined as:

$$
l_{\text {test }} \leq \frac{1}{4 f_{\max } \sqrt{L_{0} C_{0}}}=\frac{c}{4 f_{\max } \sqrt{\varepsilon_{r}}}
$$

The power cable used here was an unshielded, waterproof, PVC-insulated, fourcore cable, with a $2 \mathrm{~mm}$-diameter conductor. The relative permittivity $\left(\varepsilon_{r}\right)$ of the power cable was 3.85, so the maximum length of the test cable was $1.27 \mathrm{~m}$. In fact, it is still not accurate to estimate the cable parameters at the region where the impedance curve is near the resonance point, thus the test cable selected here was $0.67 \mathrm{~m}$ to leave enough margin, and its SC impedance curves in DM are shown in Figure 4. As seen from the impedance curves, the inductance dominates the impedance after a certain frequency, where the impedance of the cable inductor is much larger than the cable resistance, and the cable impedance curve is almost superimposed with the $20 \mathrm{~dB} /$ decade asymptote. At the same time, although the inductance varies with the frequency, the change was not as significant as the cable resistance. As a result, combined with the proposed model and the SC equivalent circuit in DM (Figure 5), two sets of impedance points located in low frequency and high frequency were enough to evaluate the model inductance parameters $\left(L_{s 1}, L_{s 2}\right)$, whereas only a part of the model resistance parameters $\left(R_{s 1}, R_{s 2}\right)$ could be solved 
using the identical sets of points and extra DC resistance $\left(R_{S C-D C}\right)$ of the cable, with the supplementary resistance parameter $\left(\Delta R_{s}\right)$ calculated by the additional point located at the natural frequency $\left(f_{R}\right)$ of the cable impedance. In general, the series branch parameters of the cable DM model could be determined by the DC resistance of the cable and three sets of points of cable impedance, including the low frequency impedance $\left(\left|Z_{S C-L F}\right|, \theta_{S C-L F}\right.$, $\left.f_{L F}\right)$, the high-frequency impedance $\left(\left|Z_{S C-H F}\right|, \theta_{S C-H F}, f_{H F}\right)$, and the natural frequency impedance $\left(\left|Z_{S C-N a}\right|, \theta_{S C-N a}, f_{\mathrm{Na}}\right)$, and their relationships are shown below:

$$
\begin{gathered}
L_{S 1}+L_{S 2}=\left|Z_{S C-L F}\right|\left[2 \pi f_{L F}\right]^{-1} \\
L_{S 1}=\left|Z_{S C-H F}\right|\left[2 \pi f_{H F}\right]^{-1} \\
R_{S 1}=R_{S C-D C} \\
R_{S 1}+R_{S 2}=\left|Z_{S C-H F}\right| \cos \theta_{S C-H F} \\
\Delta R_{S}=\left|Z_{S C-N a}\right| \cos \theta_{S C-N a}-\operatorname{Real}\left[R_{S 1}+j 2 \pi f_{N a} L_{S 1}+R_{S 2} / /\left(j 2 \pi f_{N a} L_{S 2}\right)\right]
\end{gathered}
$$
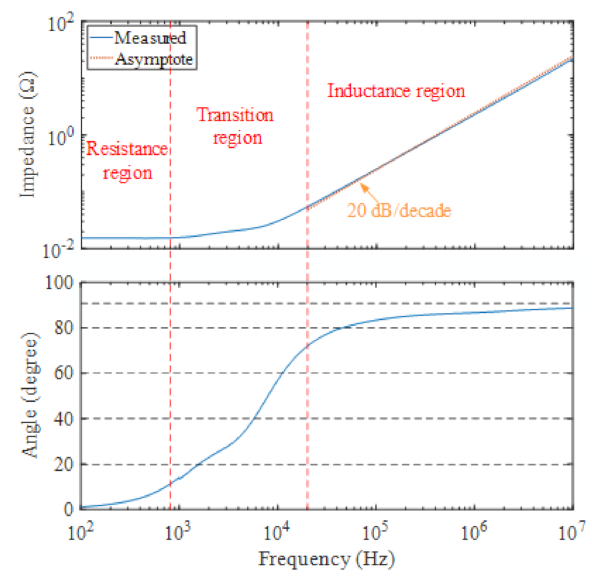

Figure 4. Short circuit (SC) impedance of the $0.67 \mathrm{~m}$ cable in DM.

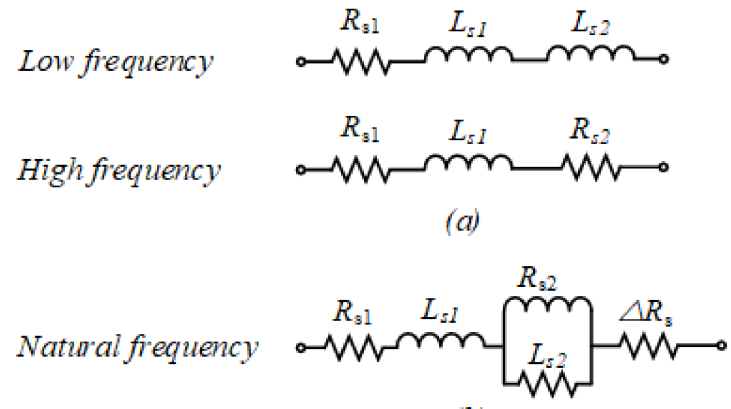

(b)

Figure 5. SC equivalent circuit at different frequency in DM.

It can be noted that the frequency in the low frequency region should be larger than the frequency point where the slope is changing from 0 to $20 \mathrm{~dB} /$ decade, rather than the $0 \mathrm{db} /$ decade region of the impedance curve. Similarly, the corresponding parallel parameters of the cable in DM can be determined from the OC impedance curve of the 0.67-m cable section, as shown:

$$
\begin{gathered}
C_{p 1}+C_{p 2}=\left[\left|Z_{O C-L F}\right|\left(2 \pi f_{L F}\right)\right]^{-1} \\
C_{p 1}=\left[\left|Z_{O C-H F}\right|\left(2 \pi f_{H F}\right)\right]^{-1} \\
R_{p 1}=R_{O C-D C}
\end{gathered}
$$




$$
\begin{gathered}
R_{p 1} / / R_{p 2}=\left|Z_{O C-H F}\right|\left[\cos \left(-\theta_{O C-H F}\right)\right]^{-1} \\
\left(\Delta R_{p}\right)^{-1}=\operatorname{Real}\left(\left|Z_{N a}\right| \angle \theta_{O C-N a}\right)^{-1}-\operatorname{Real}\left[\left(R_{p 1}\right)^{-1}+j 2 \pi f_{N a} C_{p 1}+\left(R_{p 2}+\left(j 2 \pi f_{N a} C_{p 2}\right)^{-1}\right)^{-1}\right]
\end{gathered}
$$

All the cable parameters of the DM model that were calculated based on the $0.67 \mathrm{~m}$ test cable could be transferred to per-unit parameters (Table 5), then a per-unit DM model was constructed, which was more convenient to build the long cable model. Similarly, the per-unit $\mathrm{CM}$ model could be established according to the same procedure using its corresponding measured results from the SC and OC test. In addition, the proposed model should be applied to the symmetrical cable, or a slightly asymmetric cable, depending on the degree of difference among the impedance curves of different phases.

Table 5. Per-unit parameters of the proposed model.

\begin{tabular}{ccc}
\hline Parameters & DM & $\mathbf{C M}$ \\
\hline$R_{s 1}$ & $13.5 \mathrm{~m} \Omega / \mathrm{m}$ & $15.1 \mathrm{~m} \Omega / \mathrm{m}$ \\
$R_{s 2}$ & $852.7 \mathrm{~m} \Omega / \mathrm{m}$ & $916.4 \mathrm{~m} \Omega / \mathrm{m}$ \\
$\Delta R_{s}$ & $38.8 \mathrm{~m} \Omega / \mathrm{m}$ & $22.8 \mathrm{~m} \Omega / \mathrm{m}$ \\
$R_{p 1}$ & $17.6 \mathrm{M} \Omega / \mathrm{m}$ & $15.9 \mathrm{M} \Omega / \mathrm{m}$ \\
$R_{p 2}$ & $6.4 \mathrm{k} \Omega / \mathrm{m}$ & $8.0 \mathrm{k} \Omega / \mathrm{m}$ \\
$\Delta R_{p}$ & $897.5 \mathrm{k} \Omega / \mathrm{m}$ & $840.38 \mathrm{k} \Omega / \mathrm{m}$ \\
$L_{s 1}$ & $522.3 \mathrm{nH} / \mathrm{m}$ & $432.8 \mathrm{nH} / \mathrm{m}$ \\
$L_{s 2}$ & $63.3 \mathrm{nH} / \mathrm{m}$ & $58.0 \mathrm{nH} / \mathrm{m}$ \\
$C_{p 1}$ & $86.5 \mathrm{pF} / \mathrm{m}$ & $104.5 \mathrm{pF} / \mathrm{m}$ \\
$C_{p 2}$ & $6.8 \mathrm{pF} / \mathrm{m}$ & $6.4 \mathrm{pF} / \mathrm{m}$ \\
\hline
\end{tabular}

\subsection{Impedance Verification}

Although it was discussed that the cable resistance of the proposed model at the oscillation frequency is same as the actual cable, the impedance characteristics in the high-frequency region should be verified, which is closely related to cable inductance and capacitance.

Since the proposed model is a lumped-parameter model, whose performance is intimately related to the length represented by the lumped cell, it's more accurate to use more cells to build the entire long cable model, but this takes a long simulation time. The critical length of the lumped segment depends on the rise time $\left(t_{r}\right)$ of the PWM pulse from the inverter, because the length of the lumped segment should be much smaller than the wavelength of the electromagnetic wave propagating in the cable $(\lambda)$, which can be determined by:

$$
\begin{gathered}
f_{p}=\frac{1}{\pi t_{r}} \\
\lambda=\frac{c}{\sqrt{\varepsilon_{r}}} / f_{p}
\end{gathered}
$$

In (13), $f_{p}$ denotes the highest-frequency components of a PWM pulse derived from Fourier analysis. To calculate the critical length of the test cable, the ratio of the length of the segment to the wavelength of electromagnetic wave was selected as $1.3 \times 10^{-2}$, then the critical length of the test cable was approximately $1 \mathrm{~m}$, with a $160 \mathrm{~ns}$ rise time of switching devices.

Based on the per-unit cable model and its parameters shown in Table 5, the model of a $200 \mathrm{~m}$ cable could be constructed with 200 segments, and its simulated high-frequency impedance was compared with the measured ones to verify its effectiveness. Taking the DM characteristics of the $200 \mathrm{~m}$ cable as an example (Figures 6 and 7), apparently, the frequency response based on the proposed model is in good agreement with the measured cable characteristics. 


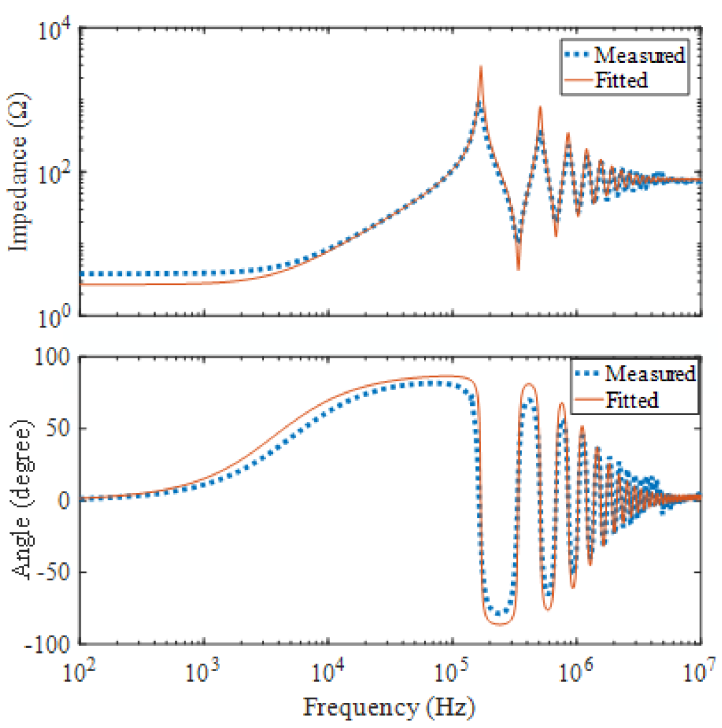

Figure 6. SC impedance of $200 \mathrm{~m}$ power cable in DM.
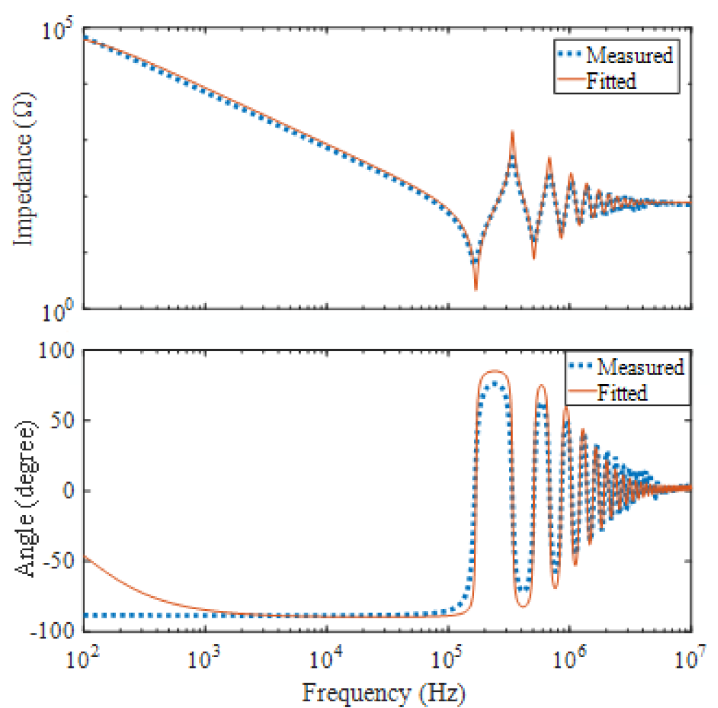

Figure 7. OC impedance of $200 \mathrm{~m}$ power cable in DM.

\section{Experimental Verification of the DM Overvoltage and CM Current}

In order to further validate the proposed modeling technique in this paper, and the predicted phenomenon based on simulation, a test platform (Figure 8) was constructed, including the $750 \mathrm{~W}$ drive system, $200 \mathrm{~m}$ cable, and $750 \mathrm{~W}$ permanent synchronous motor (PMSM). The drive system was made up of the active rectifier and the inverter, where the latter was the key factor in the drive system affecting the DM voltage and CM current, and with parameters of $380 \mathrm{~V}$ DC voltage and a $16 \mathrm{kHz}$ switching frequency. In addition, the rise time and fall time of IGBT in the inverter were approximately $0.21 \mu \mathrm{s}$ and $1.6 \mu \mathrm{s}$, respectively; these are only typical values because the switching time varied with the load. 


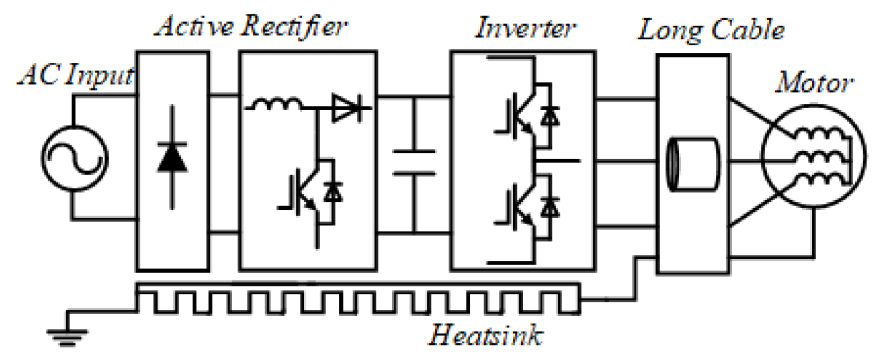

Figure 8. Experimental platform to validate the proposed model.

\subsection{Inverter and Motor Model}

The inverter and motor was also be included to make up the entire long cable system to investigate the problems caused by long cables and high switching speeds. The inverter is the voltage source of the long cable system, which could be equivalent to a voltage pulse of trapezoidal shape with a low internal impedance, or no internal impedance, since it is almost negligible compared with the characteristic impedance of the cable.

Similarly to the cable modeling, the parameters of the motors were also frequencydependent, including stator magnetizing inductance, stator leaking inductance, wingding turn-to-turn coupling capacitive, etc. There are numerous works in the literature about the high-frequency modeling of motors, but all the complex models play a limited role in most overvoltage simulations, and thus some simplifications could be adopted. The high-frequency reflection coefficient at the motor terminal was 0.95 for small hp motors, and 0.65 for $500 \mathrm{hp}$ motors, indicating that the impedance of motors is much larger than the characteristic impedance of a cable in the high-frequency region. Therefore, the small hp motors could be equivalent to an open circuit, or these could be simplified to RL circuits, even for large hp motors.

In general, the cable model is the most important and complex part of the long cable system, and the model of the inverter and motor could be simplified owing to their rather minor influence on most occasions, especially for a cable as long as, or more than, a hundred meters, where the cable model plays the main role in the aspects of DM overvoltage and $\mathrm{CM}$ current, which will be verified in the following content.

\subsection{Overvoltage Analysis}

In this section, the simulation and experimental results of line-to-line voltage at the motor side are presented to verify the feasibility of proposed system model, including the inverter, long cables, and motors. The experimental overvoltage at the motor side will be compared with that of the simulation, not only including the proposed cable model but also other cable models available in different simulator programs, like Matlab and Pspice. Figure 9 shows the simulation waveforms of the proposed cable model that are almost superimposed on the experimental counterparts, while the fitting performance of overvoltage could be further improved if the simulated output voltage of the inverter was kept the same as that of the experiment. There are some typical cable models used here for comparison with the proposed model, including the PI model of MATLAB, and TLOSSY model of Pspice, which both lack the frequency-dependant characteristics of cable parameters. From Figure 10, it can be seen that the fitting performance for overvoltage was much worse both in magnitude and damping compared with the simulation results of the proposed model. Moreover, for the high-frequency model constructed in most papers, without considering the accuracy of the cable resistance, the damping of the simulated overvoltage will be affected. In order to demonstrate such an analysis, the cable resistance of the proposed model was decreased to one fourth of its original value, and Figure 11 shows that the damping of the overvoltage waveform was not sufficient.

Based on a Bewley lattice diagram [20], if the rise time of the voltage source is less than twice the propagation time, the overvoltage at the end of cable is twice the dc-bus voltage, which is the maximum overvoltage, simultaneously. Whereas, from the overvoltage figure 
in this paper, it can be observed that the amplitude of overvoltage at the motor side was more than two times the dc-bus voltage, because the oscillation frequency was so small for very long cables that the remaining voltage at the end of the cable was still high before the application of the next voltage pulse. The overvoltage problem will be more severe if the duty ratio of the PWM is bigger, posing a big threat to the motor. Furthermore, the overvoltage will introduce a huge current spike through parasitic capacitance of the cable, which will affect the motor control and the efficiency of the entire system.
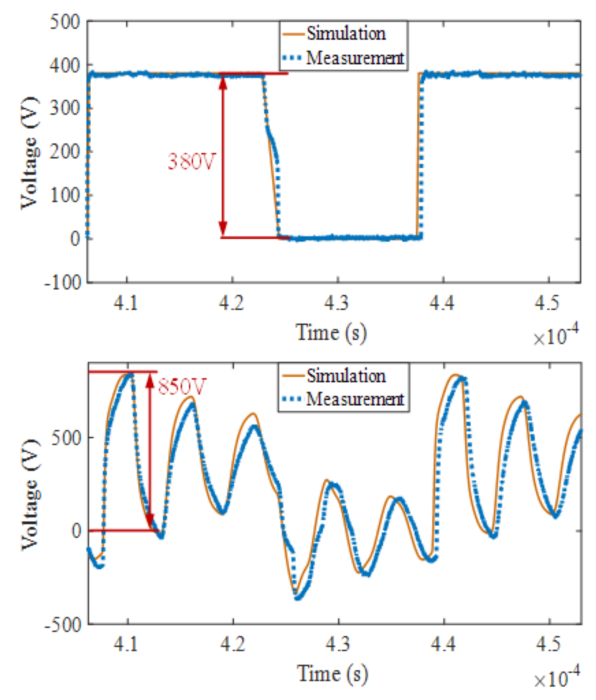

Figure 9. Comparation of the line-to-line voltage at the inverter side (upper curve) and at the motor side (bottom) with the proposed model.

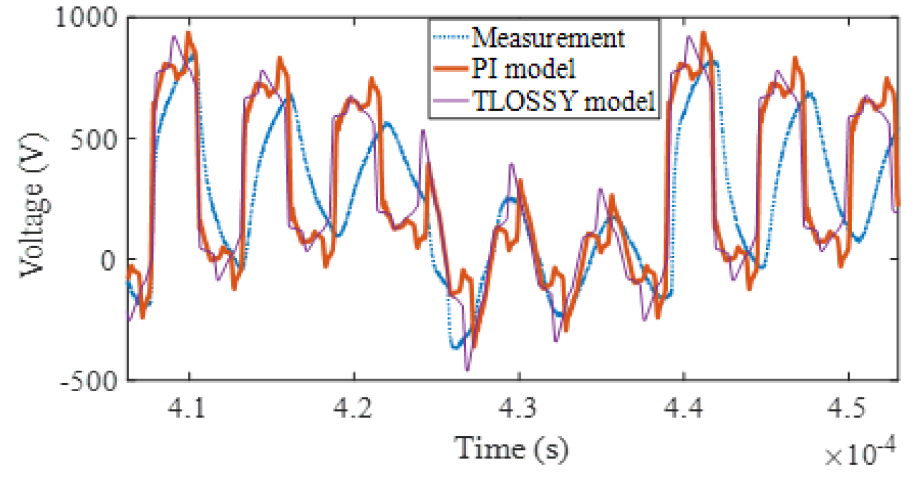

Figure 10. Comparation of the line-to-line overvoltage at the motor side with the PI model and TLOSSY model.

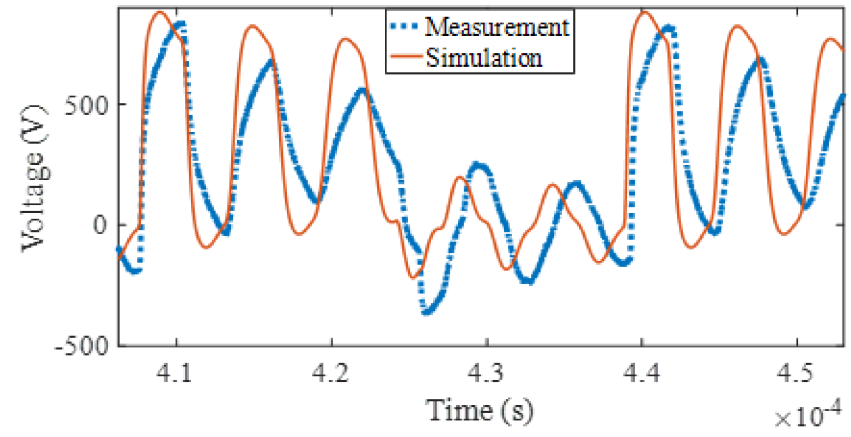

Figure 11. Comparation of the line-to-line overvoltage at the motor side with the model of low cable resistance. 


\subsection{Current Analysis}

Differently from the inverter model of the DM equivalent circuit, the CM voltage source should be one third the magnitude of the DM voltage source. The CM current analysis was carried out on the same test platform, and the measured point of the CM current was put at the beginning of the long cable to get rid of the effects of the drive system, such as the parasitic capacitance between the inverter and the ground. Compared with the DM simulation, it was hard to keep the high accuracy of the CM current simulation, because its sensitivity was affected by some trivial factors, like the capacitive coupling of the cable.

If the motor driver was directly connected with the motor through $200 \mathrm{~m}$ long cables in the test platform, the ground fault circuit interrupter (GFCI), with $30 \mathrm{~mA}$ protection threshold, responded owing to the enormous CM current (Figure 12), although consisting of high-frequency components. At the beginning of the experimental CM current, there were some small-current regions caused by the start-up strategy of motor control, like the pre-position technique. As it was shown in Figure 12, the peak value of the CM current was very high, and once the motor starts up, the power was disconnected in the response time of the GFCI. For the purpose of getting stable experimental results of the CM current with long cables to verify the simulation of the $\mathrm{CM}$ current, the length of the test cable needed to be reduced to $20 \mathrm{~m}$, whose $\mathrm{CM}$ current was not big enough to trip the protection of the GFCI. The experimental results of the $20 \mathrm{~m}$ power cable are shown in Figure 13; in which the magnitude, ring frequency, and damping of the simulation are all close to the simulation results of the proposed model, which had a better performance than the PI model and TLOSSY model. Therefore, the CM model of the drive system could be used to predict the stable CM current of the $200 \mathrm{~m}$ cable, which was not convenient to be measured in the experiment, as discussed before, demonstrating the modeling significance to some extent. It is noted that the current threshold of the GFCI protection is the rating current flowing to the ground in the low frequency range, and its protection threshold will be increased if the frequency of the ground current is higher. Nevertheless, the suppression of the enormous CM current is a big challenge in long cable systems.

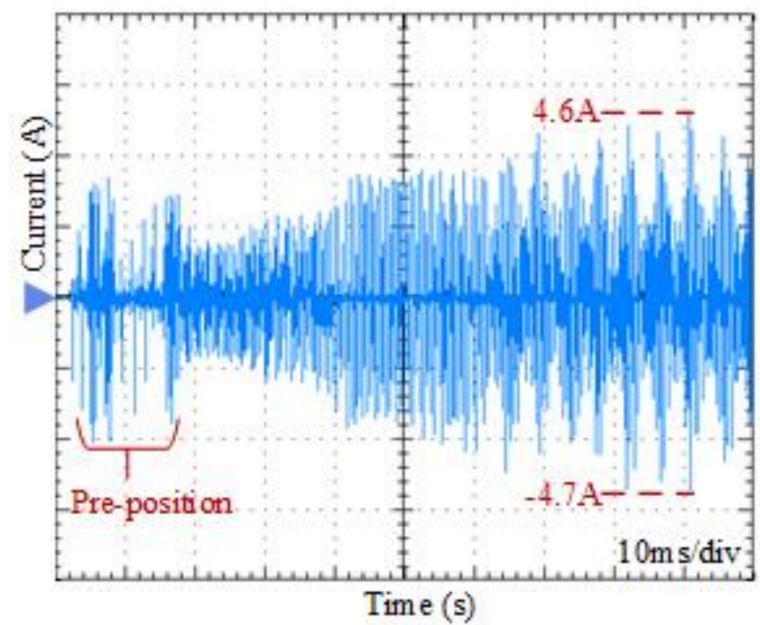

Figure 12. Measured CM current of $200 \mathrm{~m}$ cable. 

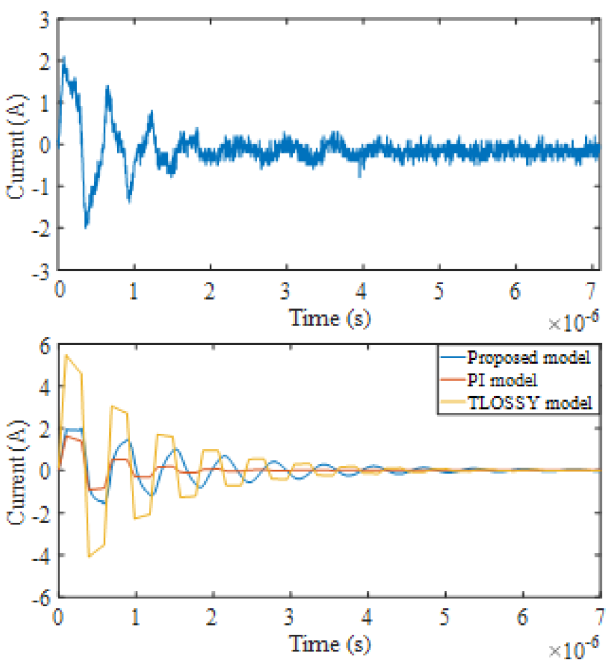

Figure 13. CM current comparison of measurement (upper curve) and simulation (bottom) for $20 \mathrm{~m}$ cable.

\section{Filter Design}

The filter design can be divided into two parts, the DM filter to suppress the overvoltage at the motor terminal and the spike current at the inverter side, and the CM filter to mitigate the $\mathrm{CM}$ current. The filters discussed here were connected to the inverter side rather than the motor side, since it is usually inconvenient to connect the filters to the motor directly.

Regarding the problems in the long cable drive system, there are some similar requirements that should be satisfied after inserting filters. Aiming at the drive system connected with the PMSM through $200 \mathrm{~m}$ cables, there are two requirements that should be met:

(1) the overvoltage at the motor side should be normally less than a designed proportion of DC voltage to guarantee the insulation and aging of motors and cables, e.g., $20 \%[18,20]$;

(2) CM current should be reduced at least to a value so that the GFCI does not trigger.

\subsection{DM Filter Design}

An output line inductor is the simplest solution to the DM overvoltage problem, but it is bulky and has a high cost. Instead, RLC and LR dv/dt filters at the inverter side have aroused some attention, in both of which the resistance $(R)$ is equal to the characteristic impedance of the cable, which will produce power dissipation as one of distinct disadvantages when filters are applied in a small power drive system. Therefore, a new LC filter with weak damping, was proposed in this paper to decrease losses but maintain good filtering performance. It should be emphasized that the design mechanism of the proposed DM filter (Figure 14) is different with that of the RLC filter, though their topologies are similar. 


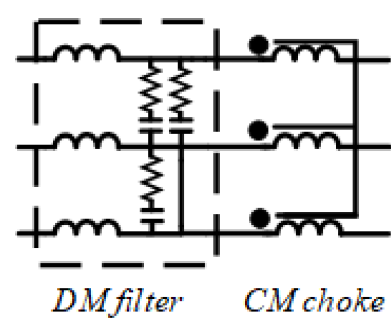

(a)

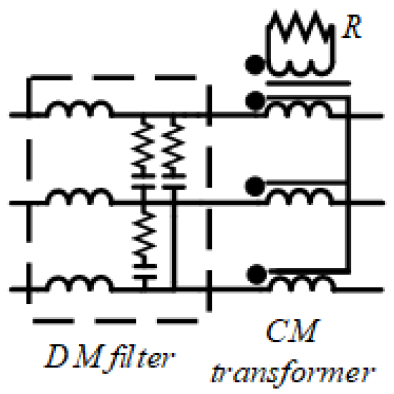

(b)

Figure 14. Proposed filter network with CM choke (a) and filter network with CM transformer (b).

As for the LC filter, its natural oscillation frequency should be far from the main frequency of the drive system, like the switching frequency of the inverter $\left(f_{P W M}\right)$ and the motor running frequency $\left(f_{\text {motor }}\right)$. Furthermore, in order to prevent the oscillation of LC filter due to disturbances from the surrounding environment, weak damping was inserted to make up the proposed filter. In other words, the proposed filter is essentially a sine-wave filter, which seems to be unnecessary and overdesigned compared to the conventional RLC and LR dv/dt filters. However, in a situation where the cable is very long and the overvoltage ratio should be low like the drive system here, the required natural oscillation frequency of the filter will be close to or even lower than the switching frequency, regardless of any sorts of filters, and therefore the merit of the proposed filter is much more distinct over the conventional RLC filter and LR filters, not only in the volume but also the losses. For a RLC filter and the proposed filter with the same inductance and capacitor, the former is worse in its ability to suppress high-frequency harmonics, which is the reason why the output voltage of the RLC filter is still PWM rather than the sinusoidal waveform, like the output voltage of the LC filter when the natural oscillation frequency of the filter is not much lower than switching frequency of inverter. The parameters of the proposed filter can be (15 18) derived from the overvoltage ratio $(\xi)$, the natural oscillation frequency $\left(f_{c}\right)$ of filter, and the damping ratio of the filter $(\zeta)$, where tr represents the required rise time of the inverter and $\Gamma_{L}$ stands for the load reflection efficient. In other words, Equations (15) and (16) are intended to make the system satisfy the voltage requirement of motors, and (17) and (18) are used to guarantee the robustness and stability of filter, particularly in avoiding oscillation of the filter.

$$
\begin{gathered}
\frac{2 l}{v t_{r}}\left(\Gamma_{L}+1\right)-1=\xi \\
f_{c}=\frac{1}{2 \pi \sqrt{L C}}<\frac{1}{\pi t_{r}} \\
10 f_{\text {Motor }}<f_{c}=\frac{1}{2 \pi \sqrt{L C}}<\frac{1}{2} f_{P W M} \\
\frac{1}{20}<\zeta=\frac{R}{2} \sqrt{\frac{C}{L}}<\frac{1}{10}
\end{gathered}
$$

According to the design criteria shown above, the parameters of proposed DM filter, designed for the drive system of test platform, were determined as

$$
L_{f_{-} D M}=427.5 \mu \mathrm{H}, C_{f_{-} D M}=0.44 \mu \mathrm{F}, R_{f_{-} D M}=3.2 \Omega
$$


Furthermore, the filter parameters of the DM equivalent circuit needed to be transferred to the per phase parameters, and the relationship of those parameters in the proposed filter is shown as

$$
L_{f_{-} p h}=\frac{2}{3} L_{f_{-} D M}, C_{f_{-} p h}=\frac{1}{2} C_{f_{-} D M}, R_{f_{-} p h}=\frac{2}{3} R_{f_{-} D M}
$$

After inserting the proposed DM filter, the voltage at motor side was sinusoidal, which was apparently better than the output waveform of the RLC filter using the same inductance and capacitance, as shown in Figure 15. In Table 6, there is a comparison between the proposed filter $\left(L_{f_{-} p h}=285 \mu \mathrm{H}, C_{f_{-} p h}=0.22 \mu \mathrm{F}, R_{f_{-} p h}=2 \Omega\right)$, RLC filter $\left(L_{f_{-} p h}=285 \mu \mathrm{H}\right.$, $\left.C_{f_{-} p h}=0.22 \mu \mathrm{F}, R_{f_{-} p h}=50 \Omega\right)$, and LR filter $\left(L_{f_{-} p h}=285 \mu \mathrm{H}, R_{f_{-} p h}=50 \Omega\right)$, which shows the advantage of the proposed filter from the perspective of volume, efficiency, and suppression ability of overvoltage. In addition, the high-frequency losses of the cables and motors were also eliminated by implementing the proposed filter, because the output voltage of the proposed filter is sinusoidal. In general, the proposed filter has a high efficiency, smaller volume, and lower overvoltage.
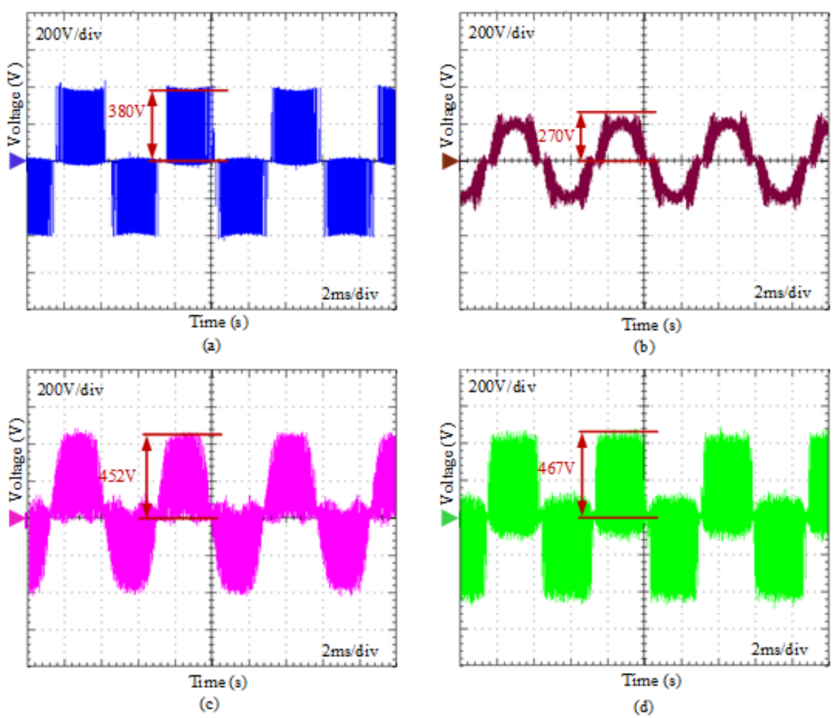

Figure 15. Output voltage of inverter (a) and terminal voltage at motor side with the proposed DM filter (b), RLC filter (c), LR filter (d).

Table 6. Comparison between proposed filter and conventional filters.

\begin{tabular}{cccc}
\hline & Proposed Filter & RLC Filter & LR Filter \\
\hline Number of Components & 9 & 9 & 6 \\
Volume & small & big & small \\
Overvoltage & none & $19 \%$ & $23 \%$ \\
Filter losses & $20 \mathrm{~W}$ & $290 \mathrm{~W}$ & $135 \mathrm{~W}$ \\
\hline
\end{tabular}

\subsection{Filter Design}

The sources of the $\mathrm{CM}$ voltage in the entire drive system are the rectifier and inverter, and the latter plays the main role. For the convenience of analysis, the modulation of inverter was simplified as the SPWM (Sinusoidal Pulse Width Modulation), and the midpoint of DC side was selected as reference potential point, shown in the Figure 16. Then, the common mode voltage $\left(V_{c m 1}\right)$ at the inverter side could be derived as follows:

$$
V_{i O}=S_{i} \frac{U_{d c}}{2}+\left(1-S_{i}\right)\left(-\frac{U_{d c}}{2}\right)=\left(2 S_{i}-1\right) \frac{U_{d c}}{2}(i=U, V, W)
$$




$$
V_{c m 1}=\frac{V_{U O}+V_{V O}+V_{W O}}{3}=\frac{U_{d c}}{6} \sum_{i=U, V, W}\left(2 S_{i}-1\right)
$$

where $S_{i}$ is the switching function, if the upper switch of $i$-phase is on, and the function value is 1 , otherwise it is 0 . Considering the overvoltage ratio $(n)$ at the motor side with long cables and assuming the voltage applied on the long cables is symmetrical, the $\mathrm{CM}$ voltage at the motor side can be approximately expressed as:

$$
V_{c m 1}^{\prime}=\frac{V_{U^{\prime} O}+V_{V^{\prime} O}+V_{W^{\prime} O}}{3}=\frac{n U_{d c}}{6} \sum_{i=U, V, W}\left(2 S_{i}-1\right)
$$

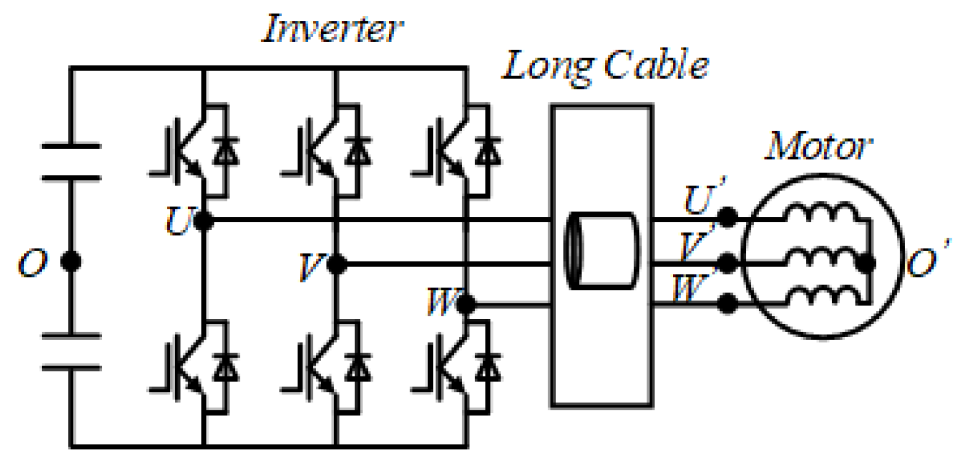

Figure 16. Simplified drive system used to analyze the CM noise.

In fact, the midpoint $(\mathrm{O})$ voltage of the dc-bus side is not equal to the ground $(\mathrm{N})$, and the value of difference $\left(V_{O N}\right)$ depends on the topology of the rectifier, which is actually a $\mathrm{CM}$ voltage source caused by the rectifier.

$$
V_{c m 2}=V_{O N}
$$

For the uncontrolled rectifier or the single active rectifier used here, whose $\mathrm{CM}$ source voltage is mainly made up of low frequency components, their high-frequency CM noise was much smaller than that of the inverter. In general, the nonlinear components were the $\mathrm{CM}$ source, which could be regarded as the voltage source in the CM equivalent circuit, as shown in Figure 17. In the $\mathrm{CM}$ equivalent circuit, $C_{p}$ denotes the parasitic capacitance, including the rectifier, inverter, and motor to ground, and $L_{G N D}$ represents the ground inductance of the ground path. Additionally, the arrows of current do not imply the actual flowing direction, and the $\mathrm{CM}$ current flowing out of the drive system can be expressed as:

$$
i_{c m}=i_{c m 3}-\left(i_{c m 1}+i_{c m 2}\right)
$$

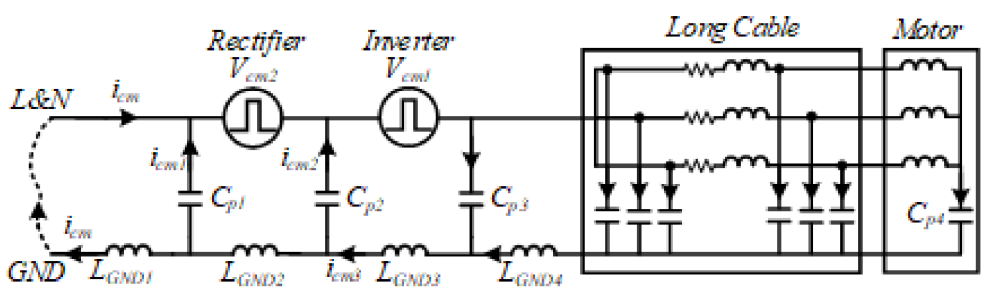

Figure 17. Equivalent CM path of the drive system.

Therefore, in order to decrease the value of the CM current $\left(i_{c m}\right)$ flowing out the drive system, the values of $i_{c m 1}$ and $i_{c m 2}$ should be increased, e.g., through paralleling capacitance before the $\mathrm{CM}$ source, or adding $\mathrm{CM}$ inductance at the $\mathrm{AC}$ side, and the value of $i_{\mathrm{cm} 3}$ should be decreased, e.g., through adding a $\mathrm{CM}$ filter after the inverter. As for the $\mathrm{CM}$ filter at the inverter side, if using the $\mathrm{CM}$ choke, the saturation current should be high enough due to the large CM current caused by long cables. The inductance of the CM choke designed in 
the test platform was $10 \mathrm{mH}$, with a rated saturation current of $0.2 \mathrm{~A}$. However, the $\mathrm{CM}$ transformer (Figure 15) is an alternative choice to decrease the volume of the CM filter, which requires a lower saturation current almost without adding cost. Compared with the $\mathrm{CM}$ choke, the $\mathrm{CM}$ transformer introduced a damping resistor to mitigate the oscillation of $\mathrm{CM}$ current and decrease its peak value. The theoretical design of damping resistance has been clarified in [21], which could be verified ahead of the experiment, based on the constructed model of the drive system. After inserting the CM filter, the CM current was extremely small, thus the dissipation on the damping resistor was very small and the area of secondary winding could be thin, contributing to the priority of the CM transformer combined with a smaller magnetic core compared with the CM choke. In the experiment, a $\mathrm{CM}$ transformer with $4.3 \mathrm{mH}$ inductance and $2500 \Omega$ was inserted, and the corresponding CM current waveform is shown in Figure 18, where the CM current was greatly decreased compared with that in Figure 12. In addition, the loss of the CM filter was about $6.25 \mathrm{~W}$; therefore, the total loss of the proposed filter in Figure $15 b$ was $26.25 \mathrm{~W}$.

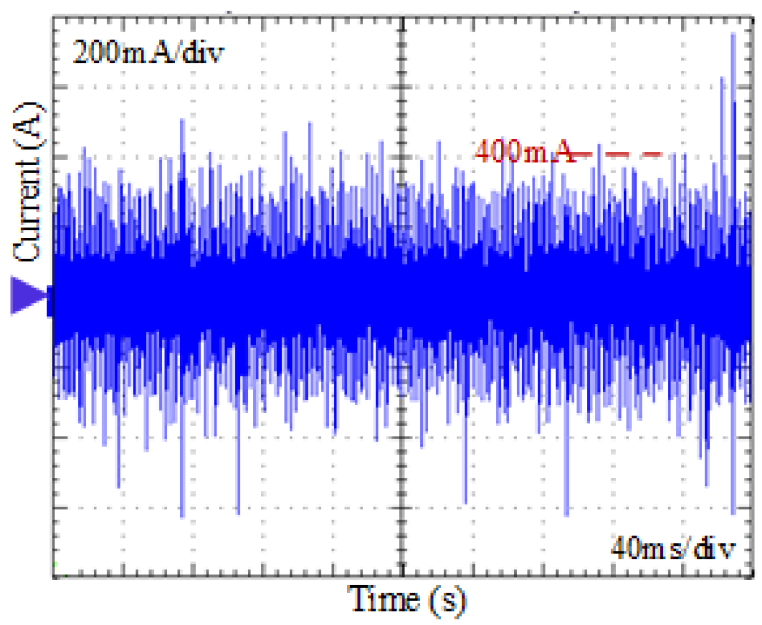

Figure 18. CM current of $200 \mathrm{~m}$ cable after inserting the CM transformer.

\section{Conclusions}

This paper analyzed the importance of the high-frequency resistance of a cable, which is an easily overlooked factor in the process of cable modeling. Then, both high-frequency models were proposed to accurately describe the characteristics of power cables in a wide frequency range, but the ladder model required enormous calculation, and it took more time to simulate the model. The model parameters were identified through the DM and CM impedance characteristics measured by the impedance analyzer, with analytical design equations given in this paper. Combined with the simplified inverter model and motor model, the model of the whole drive system was constructed to predict the DM overvoltage and $\mathrm{CM}$ current. The simulation results were in good agreement with the experimental waveform, indicating the effectiveness of the proposed model.

Considering the high losses of conventional DM filters, a new filter at the motor side was proposed to mitigate the DM overvoltage. The new DM filter was validated with experiments and compared with two sorts of conventional DM filters, showing the advantage of the proposed DM filter in aspects of volume, losses, and suppressing ability. For the suppression of $\mathrm{CM}$ current, a $\mathrm{CM}$ choke and $\mathrm{CM}$ transformer were adopted in this paper, either of which forms the entire filter network with the proposed DM filter. The filter networks were more effective in the small power drive system with long cables and motors that requires a strict working voltage. 
Author Contributions: Conceptualization, L.W.; methodology, L.W.; software. L.W.; validation, Y.Z. and M.S.A.; formal analysis, L.W.; investigation, G.C. and J.M.G.; data curation, L.W.; writingoriginal draft preparation, L.W.; writing_review and editing, G.C. and J.M.G.; supervision, J.C.V., J.M.G. and G.C.; funding acquisition, J.M.G. and G.C. All authors have read and agreed to the published version of the manuscript.

Funding: This work was supported by National Natural Science Foundation of China (\#51777186) and VILLUM FONDEN under the VILLUM Investigator Grant (No. 25920): Center for Research on Microgrids (CROM); www.crom.et.aau.dk (accessed on 18 February 2021).

Institutional Review Board Statement: Not applicable.

Informed Consent Statement: Not applicable.

Data Availability Statement: Not applicable.

Conflicts of Interest: The authors declare no conflict of interest.

\section{References}

1. Zhang, Z.; Wang, F.; Tolbert, L.M.; Blalock, B.J.; Costinett, D. Evaluation of switching performance of SiC devices in PWM inverter fed induction motor drives. IEEE Trans. Power Electron. 2014, 30, 1597-1604.

2. Smochek, M.; Pollice, A.F.; Rastogi, M.; Harshman, M. Long cable applications from a medium-voltage drives perspective. IEEE Trans. Ind. Appl. 2016, 52, 645-652. [CrossRef]

3. De Paula, V.C.; De Paula, H. Employing DC transmission in long distance AC motor drives: Analysis of the copper economy and power losses reduction in mining facilities. In Proceedings of the IEEE Industry Application Society-51st Annual Meeting, IAS 2015, Conference Record, Addison, TX, USA, 18-22 October 2015.

4. Song, Y.; Ebrahimzadeh, E.; Blaabjerg, F. Analysis of High-Frequency Resonance in DFIG-Based Offshore Wind Farm via Long Transmission Cable. IEEE Trans. Energy Convers. 2018, 33, 1036-1046. [CrossRef]

5. Jiang, Y.; Wu, W.; He, Y.; Chung, H.S.H.; Blaabjerg, F. New Passive Filter Design Method for Overvoltage Suppression and Bearing Currents Mitigation in a Long Cable Based PWM Inverter-Fed Motor Drive System. IEEE Trans. Power Electron. 2017, 32, 7882-7893. [CrossRef]

6. Mirafzal, B.; Skibinski, G.L.; Tallam, R.M. Determination of parameters in the universal induction motor model. IEEE Trans. Ind. Appl. 2009, 45, 142-151. [CrossRef]

7. Magdun, O.; Binder, A.; Purcarea, C.; Rocks, A. High-frequency induction machine models for calculation and prediction of common mode stator ground currents in electric drive systems. In Proceedings of the 2009 13th European Conference on Power Electronics and Applications EPE '09, Barcelona, Spain, 8-10 September 2009.

8. Pulsinelli, F.; Solero, L.; Chiola, D.; Sobe, K.; Brucchi, F. Overvoltages at Motor Terminals in SiC Electric Drives. In Proceedings of the 2018 International Symposium on Power Electronics, Electrical Drives, Automation and Motion (SPEEDAM), Amalfi, Italy, 20-22 June 2018; pp. 513-518.

9. Stevanovic, I.; Wunsch, B.; Skibin, S. Behavioral high-frequency modeling of electrical motors. In Proceedings of the 2013 Twenty-Eighth Annual IEEE Applied Power Electronics Conference and Exposition (APEC), Long Beach, CA, USA, 17-21 March 2013; Volume 2, pp. 2547-2550.

10. Schinkel, M.; Weber, S.; Guttowski, S.; John, W.; Reichl, H. Efficient HF modeling and model parameterization of induction machines for time and frequency domain simulations. In Proceedings of the Twenty-First Annual IEEE Applied Power Electronics Conference and Exposition APEC, Dallas, TX, USA, 19-23 March 2006; Volume 2006, pp. 1181-1186.

11. Moreira, F.A.; Martí, J.R.; Zanetta, L.C. Latency techniques applied to the transient simulation of transmission lines using the Z-Line Model. In Proceedings of the 2006 IEEE/PES Transmission \& Distribution Conference and Exposition: Latin America TDC'06, Caracas, Venezuela, 15-18 August 2006; pp. 1-6.

12. Boglietti, A.; Carpaneto, E. Induction motor high frequency model. In Proceedings of the Conference Record-IAS Annual Meeting (IEEE Industry Applications Society), Phoenix, AZ, USA, 3-7 October 1999.

13. Weber, S.P.; Hoene, E.; Guttowski, S.; John, W.; Reichl, H. Modeling induction machines for EMC-analysis. In Proceedings of the 2004 IEEE 35th Annual Power Electronics Specialists Conference, Aachen, Germany, 20-25 June 2004; Volume 1, pp. 94-98.

14. De Paula, H.; de Andrade, D.A.; Chaves, M.L.R.; Domingos, J.L.; de Freitas, M.A.A. Methodology for cable modeling and simulation for high-frequency phenomena studies in PWM motor drives. IEEE Trans. Power Electron. 2008, 23, 744-752. [CrossRef]

15. Skibinski, G.; Leggate, D.; Kerkman, R. Cable characteristics and their influence on motor over-voltages. In Proceedings of the APEC 97-Applied Power Electronics Conference, Atlanta, GA, USA, 27 February 1997; Volume 1, pp. 114-121.

16. Skibinski, G.; Tallam, R.; Reese, R.; Buchholz, B.; Lukaszewski, R. Common mode and differential mode analysis of three phase cables for PWM AC drives. In Proceedings of the Conference Record of the 2006 IEEE Industry Applications Conference Forty-First IAS Annual Meeting, Tampa, FL, USA, 8-12 October 2006; Volume 2, pp. 880-888.

17. Akagi, H.; Matsumura, I. Overvoltage mitigation of inverter-driven motors with long cables of different lengths. IEEE Trans. Ind. Appl. 2011, 47, 1741-1748. [CrossRef] 
18. Rendusara, D.A.; Enjeti, P.N. An improved inverter output filter configuration reduces common and differential modes dv/dt at the motor terminals in PWM drive systems. IEEE Trans. Power Electron. 1998, 13, 1135-1143. [CrossRef]

19. Moreira, A.F.; Lipo, T.A.; Venkataramanan, G.; Bernet, S. High-frequency modeling for cable and induction motor overvoltage studies in long cable drives. IEEE Trans. Ind. Appl. 2002, 38, 1297-1306. [CrossRef]

20. Tallam, R.M.; Member, S.; Skibinski, G.L.; Shudarek, T.A.; Lukaszewski, R.A.; Member, S. Integrated Differential-Mode and Common-Mode Filter to Mitigate the Effects of Long Motor Leads on AC Drives. IEEE Trans. Ind. Appl. 2011, 47, 2075-2083. [CrossRef]

21. Ogasawara, S. Modeling and damping of high-frequency leakage currents in PWM inverter-fed AC motor drive systems. IEEE Trans. Ind. Appl. 1996, 32, 1105-1114. [CrossRef]

22. Wang, L.; Ho, C.N.M.; Canales, F.; Jatskevich, J. High-frequency modeling of the long-cable-fed induction motor drive system using TLM approach for predicting overvoltage transients. IEEE Trans. Power Electron. 2010, 25, 2653-2664. [CrossRef] 